\title{
Novel thermal method for determining properties of compressed natural gas
}

The article describes a new method for obtaining fuel properties, derived from the thermal properties of natural gas fuel mixtures. By measuring the thermal conductivity and the dynamic response with the help of a heated Micro Electro Mechanical System (MEMS), input values for a mathematical correlation are obtained which predict the calorific value of the gas. In this paper the fundamentals of the theoretical gas properties, the sensor operation and the first results on a gas test bench are presented.

Key words: calorific value, methane number, heat capacitance, temperature conductance, natural gas quality

\section{Introduction}

The composition of compressed natural gas $(\mathrm{CNG})$ varies strongly worldwide. Even across Europe the gas blends differ according to the gas source location. Additional to the impacts of its origin, the manufacturing process plays a role in the content of the hydrocarbons methane, ethane, propane and butane as well as the inert gases carbon monoxide and nitrogen, which are the main fractions of hydrocarbons in CNG. New fuel trends like bio-methane, power to gas $(\mathrm{PtG})$ methane and hythane (blends of hydrogen and natural gas) can lead to a further diversification of the gaseous combustion media.

According to the big variances the quality of the natural gas variation, which can be classified with the calorific value $(\mathrm{CV})$ and the methane number $(\mathrm{MN})$. This issue is specific to natural gas and has a strong influence on the engine control optimization capability. For a diagnostic and anticipatory engine operation the methane number and calorific value can be relevant optimization parameter. If an automotive sensor which delivers $\mathrm{CV}$ and $\mathrm{MN}$ can be developed, then ignition timing can be adjusted to operate the engine close to the knock line. With the calorific value it is possible to optimize ignition angle and/or boost pressure. In addition, the calorific value can play an important role for achieving gas-only, mono-fuel-engines. A sensor signal, provided to the engine management system in the time frame of one second before engine on, would avoid rich mixture combustion and potential critical engine crank-up conditions, especially just after the gas refilling operation.

Existing ways of measuring the quality of the natural gas like calorimetry can't be used, are too complex or do not work in the relevant temperature and pressure range. A novel method with a simple microsystem chip is the starting point for a concept study to get information from natural gas blends by measuring thermal properties. The soderived thermal properties are the inputs for a mathematical correlation model which should predict the calorific value and methane number.

\section{Natural gas variety}

Defining the quality of fuels, oils and other complex fluid mixtures is a difficult topic. The presence and variation of single compounds affect the properties of the fuel in many ways. Compressed natural gas blends can contain hydrocarbons, inert gases, sulfur components, hydrogen, water and odour gases. Of course, methane is the dominant fraction. The hydrocarbons ethane, propane and butane play a role in the percentage range. The inert gases carbon dioxide and nitrogen with a possible content of several percent have also a significant impact. An example how the content of the main components can vary over different locations is given in [6].

Table 1. Main components of natural gas blends at different locations [6]

\begin{tabular}{|c|c|c|c|c|c|}
\hline \multirow{2}{*}{ Gas } & \multicolumn{3}{|c|}{ Natural Gasoline $\mathrm{H}$} & \multicolumn{2}{c|}{$\begin{array}{c}\text { Natural Gasoline L } \\
\text { [mol\%] }\end{array}$} \\
\cline { 2 - 6 } & Russia & $\begin{array}{c}\text { North } \\
\text { Sea }\end{array}$ & Denmark & Holland & Germany \\
\hline $\mathrm{CH}_{4}$ & 96.96 & 88.71 & 90.07 & 84.64 & 86.46 \\
\hline $\mathrm{C}_{2} \mathrm{H}_{6}$ & 1.37 & 6.93 & 5.68 & 3.56 & 1.06 \\
\hline $\mathrm{C}_{3} \mathrm{H}_{6}$ & 0.45 & 1.25 & 2.19 & 0.61 & 0.11 \\
\hline $\mathrm{C}_{4} \mathrm{H}_{10}$ & 0.015 & 0.28 & 0.90 & 0.19 & 0.03 \\
\hline larger $\mathrm{HCs}$ & 0.03 & 0.07 & 0.28 & 0.13 & 0.01 \\
\hline $\mathrm{N}_{2}$ & 0.86 & 0.82 & 0.28 & 10.21 & 10.24 \\
\hline $\mathrm{CO}_{2}$ & 0.18 & 1.94 & 0.60 & 1.68 & 2.08 \\
\hline
\end{tabular}

Some other gases like hydrogen, oxygen and long chained hydrocarbons are in a typical range of a few percent or less than $1 \%$ in concentration. For the generic study of a thermal quality sensor this small fractions are neglected so far.

Table 2. Selected gas species and relevant measurement ranges

\begin{tabular}{|l|c|c|}
\hline Gas compound & $\begin{array}{c}\text { Minimum range } \\
{[\mathrm{vol} \%]}\end{array}$ & $\begin{array}{c}\text { Maximum range } \\
{[\mathrm{vol} \%]}\end{array}$ \\
\hline Methane $\mathrm{CH}_{4}$ & 70 & 100 \\
\hline Ethane $\mathrm{C}_{2} \mathrm{H}_{6}$ & 0 & 20 \\
\hline Propane $\mathrm{C}_{3} \mathrm{H}_{8}$ & 0 & 15 \\
\hline Butane $\mathrm{C}_{4} \mathrm{H}_{10}$ & 0 & 10 \\
\hline Nitrogen $\mathrm{N}_{2}$ & 0 & 30 \\
\hline Carbondioxid $\mathrm{CO}_{2}$ & 0 & 20 \\
\hline
\end{tabular}

To cover the worldwide variations of $\mathrm{CNG}$, detecting the variability at fuel refilling, providing an instantaneous measurement before engine start, adjusting the efficiency of 
combustion and controlling knock resistance the calorific value and the methane number are the desired values for characterization. The target for the sensor concept proof is to measure $\mathrm{MN}$ and $\mathrm{CV}$ as accurate as possible.

In the quality sensor evaluation of work package 5 the investigations are restricted to gases which are in the percentage range. The physical properties used for the sensing of the gas mixture are dominated by the main fractions. A limitation on six $\mathrm{CNG}$ gas fractions with methane, ethane, propane, pentane, carbon dioxide and nitrogen seem to reflect the nature of the gas in an appropriate way. They allow adjusting a continuous composition for detailed investigations.

\section{Lower calorific value and methane number}

The lower calorific value (LCV) describes the whole redundant energy when a volume of gaseous fuels is completely burnt and water vapor leaves with the combustion products without being condensed. Because many gas burning applications cannot make use of the heat content of the water vapor, the lower calorific value is the more interesting one. It is essential for driving range estimation based on the calculated tank filling level [3].

$$
\mathrm{LCV}_{\mathrm{m}}=\sum_{\mathrm{n}} \mathrm{x}_{\mathrm{n}} \cdot \mathrm{LCV}_{\mathrm{m}, \mathrm{n}}
$$

The methane number $(\mathrm{MN})$ is an indicator for the knocking resistance of gaseous fuels, similar to the octane number of gasoline vehicles. The $\mathrm{MN}$ of a gaseous mixture is defined as the methane volume percentage of a pure combination of only methane and hydrogen, which has the same knocking resistance. The methane number was determined experimentally on test engines at AVL in the seventies and now standardized in DIN51624 [8].

\section{Thermal properties as basis for gas quality prediction}

It is difficult to get e.g. the calorific value without combusting the gas and simultaniously measureing the heat release. A direct measurement is not possible in a simple way. The novel approach is now to measure the thermal conductivity and specific heat capacity with a micromachined and miniaturized sensor. The typical specific thermal properties of each single gas in a CNG blend allow to destinguish concentrations in mixtures by measuring and tracking the thermal properties. In combination with a mathematic correlation model the prediction of gas quality properties is possible. As signal for the calculation input the thermal conductivity and the specific heat capacity is used.

Both physical values are temperature dependent. With a dedicated operation strategy of the MEMS device this temprature dependency can be exploited by measuring at different test gas temperatures. As a consequence an additional input parameter for the correlation modeling can be adapted. This widening of the input parameter is the key for a more detailed and accurate modeling taking the temperature dependencies into consideration.

\subsection{Thermal conductivity of a single gas}

Thermal conduction is the transfer of heat from hotter to cooler parts of a medium resulting in temperature equilibrium. Taking gaseous fluids into consideration, the thermal conductivity is the energy transfer between gas molecules from higher to ones with lower energy. The basic law of thermal conductivity is the Fourier Law which states that the heat flux density is proportional to the temperature gradient $\mathrm{T}$ in an isotropic medium [4]:

$$
\mathrm{q}=\frac{\mathrm{dQ}}{\mathrm{A} * \mathrm{dt}}=-\lambda \frac{\mathrm{dT}}{\mathrm{dz}}
$$

The proportional factor $\lambda$ is the thermal conductivity. The minus indicates that the temperature decreases in the direction of the heat transfer [6]. The absolute temperature has an impact on the lambda value. An approximation for the temperature dependency of the thermal conductivity is described in the VDI Wäremeatlas [7] with an 4th polynomial equation and the gas specific coefficient A,B,C,D and E.

$$
\frac{\lambda}{\mathrm{W} / \mathrm{K} \cdot \mathrm{m}}=\mathrm{A}+\mathrm{B} \frac{\mathrm{T}}{\mathrm{K}}+\mathrm{C}\left(\frac{\mathrm{T}}{\mathrm{K}}\right)^{2}+\mathrm{D}\left(\frac{\mathrm{T}}{\mathrm{K}}\right)^{3}+\mathrm{E}\left(\frac{\mathrm{T}}{\mathrm{K}}\right)^{4}
$$

The characteristics over the application temperature range for the CNG relevant gases is displayed in Fig. 1.

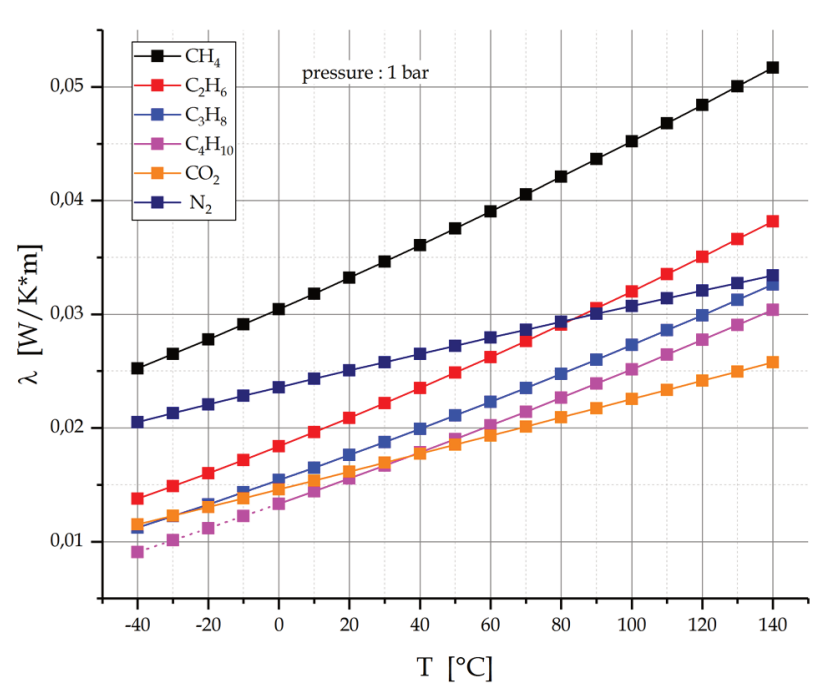

Fig. 1. Temperature dependency of the thermal conductivity $\lambda$ for different pure gases

The overview shows the value of $\lambda$ differs for each pure gas species at a dedicated temperature as well as the slope, taking the temperature influence into count.

\subsection{Specific heat capacity at constant pressure for pure gases}

The heat capacity of each medium tells us how much heat is required to raise a certain amount of it by one degree. For gases we can define a molar heat capacity, which is the heat required to increase the temperature of 1 mole of the gas by $1 \mathrm{~K}$.

$$
\mathrm{Q}=\mathrm{nC} \cdot \Delta \mathrm{T}
$$


The value of the heat capacity depends on, wheater the heat is added at constant volume or constant pressure. Therefore, the specific heat capacity $\mathrm{Cp}$ at constant pressure is:

$$
\mathrm{Cp}=\frac{\Delta \mathrm{Q}}{\Delta \mathrm{T}}
$$

Where accoding to the first law of thermodynamics some of the heat energy is doing work due to constant pressure [1]. The value for $\mathrm{Cp}$ is tempeature dependent. Like before described in the thermal conductivity section, the characteristics can be similar expressed by a interpolation equation with the coeffcients A, B, C, D and E [7].

$$
\frac{\mathrm{c}_{\mathrm{p}}}{\mathrm{J} / \mathrm{kg} \cdot \mathrm{K}}=\mathrm{A}+\mathrm{B} \frac{\mathrm{T}}{\mathrm{K}}+\mathrm{C}\left(\frac{\mathrm{T}}{\mathrm{K}}\right)^{2}+\mathrm{D}\left(\frac{\mathrm{T}}{\mathrm{K}}\right)^{3}+\frac{\mathrm{E}}{(\mathrm{T} / \mathrm{K})^{2}}
$$

One can see in Fig. 2 the specific heat capacity of the inert gases carbon dioxide and nitrogen in comparsion to the main hydrocarbon fractions in the relevant temperature range.

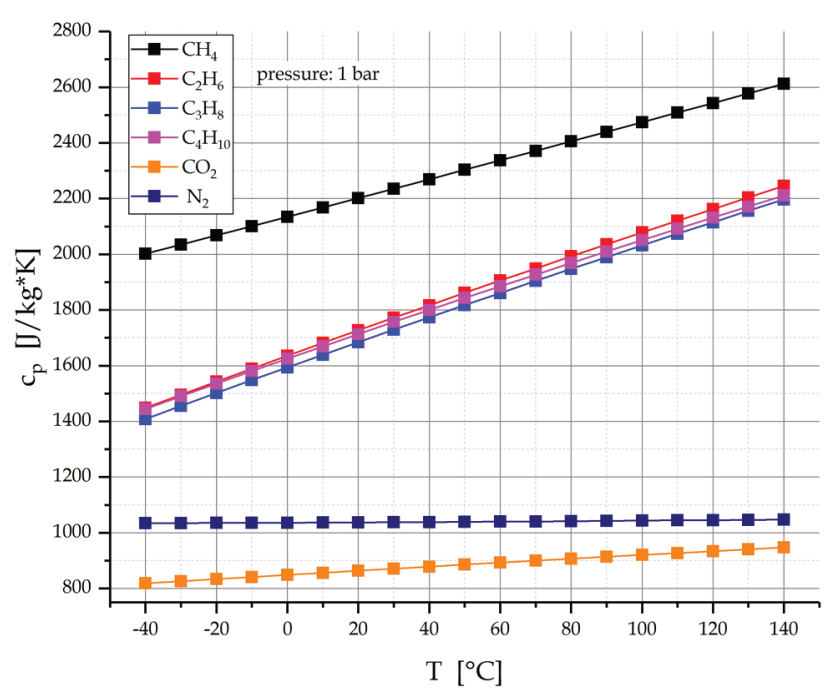

Fig. 2. Heat capacity at constant pressure for different gases and temperatures

The following content, can be derived from the data shown in Fig. 4. Methan has by far the highest heat capacity. The other hydrocarbons ethane, propane and butane are almost identical in value and slope. For the inert gases nitrogen and carbon dioxide the specific heat capacity values are much lower and the progression over temperatue, is almost constant, especially for nitrogen.

\subsection{Time dependent temperature distribution}

Another physical relation which discribes the time dependent temperature distribution is the so called thermal diffusivity. The thermal conductivity $\lambda$, the coefficient of heat capacity $c$ and the density $\rho$ of a substance can be summarized in absence of internal heat sources by the differential equation of thermal conductivity [5]:

$$
\frac{\partial \mathrm{T}}{\partial \tau}=\frac{\lambda}{\rho c} \cdot \nabla^{2} \cdot \mathrm{T}
$$

$$
\nabla^{2} \cdot \mathrm{T}=\frac{\partial^{2} \cdot \mathrm{T}}{\partial \cdot \mathrm{x}^{2}}+\frac{\partial^{2} \cdot \mathrm{T}}{\partial \cdot \mathrm{y}^{2}}+\frac{\partial^{2} \cdot \mathrm{T}}{\partial \cdot \mathrm{z}^{2}}
$$

With $\nabla$ the Laplacian operator, $\tau$ the time, and $x, y, z$ the cartesian coordinates. Wheras the group $k=(\lambda / \rho c)$ is known as thermal diffusivity. It characterizes the velocity of propagation of isothermal surfaces in a body.

In the study the specific heat capacity is taken as indicator or so to say deceicive propertie for the time dependent temperature change. The use of the thermal diffusivity would also be possible, but the operation of the sensor described later on is in contradiction to the absence of an internal heat source.

Although the pure specific heat capacity does not fully reflect the physical circumstances, the results achieved are sufficent.

\subsection{Thermal properties of gas mixtures}

The thermal properties of gas mixtures can be summarized by the properties of the single gases. The following two formulas are used for the theoretical calculations and the comparsion with the measurements achieved.

For the thermal conductivity the mixing equations of Wassiljeva, Ason and Saxena can be used. The indice $i$ and $\mathrm{j}$ representing components according to the single gase. In the equation $\mathrm{x}$ stands for the quantity and $\mathrm{F}$ is an own gas specific summary, considering the viscosity gas and molare weight of the gas [2].

$$
\lambda^{\mathrm{Gem}}=\sum_{\mathrm{i}} \frac{\mathrm{x}_{\mathrm{i}} \cdot \lambda_{\mathrm{i}}}{\sum_{\mathrm{j}} \mathrm{x}_{\mathrm{j}} \cdot \mathrm{F}_{\mathrm{ij}}}
$$

For the calculation of the specfic heat capacity of an ideal gas only the massfraction of the heat capcity are summed up [7].

$$
c_{p, G e m}=\sum_{i} x_{i} \cdot c_{p, i}
$$

For the calculation of the specfic heat capacity of an ideal gas only the mass fraction of the heat capacity are summed up. These two mixing formulas are used for theoretical approximations.

\section{Sensor properties}

Typical sensor arrangenments for measuring the thermal properties of gases are micromachined membranes. They have the required sensitivity to destinguish small changes of e.g. the thermal conductivity. Typical for this membranes are silicon nitride processed layers, made in bulk micromachining technology to achieve the well thermally isolated measurement with a measurement spot on top of the membrane. An even more advanced setup shown in Fig. 3 is used for the CNG based measurement.

In the considered implemention the membane is not a fully closed one, etched from the backside. In the used device it is in fact a membrane layer processed in surface micromachined technology on top of the semiconducter which is opened due to an underetching process on two sides. A suspended bridge with heater structure on top remains as sensitive device. This allowes an even better 
thermal isolation of the gas sensitive bridge from the solid state silicon substrate. The area which is responsible for the heat transfer is in the range of $\sim 1 \mathrm{~mm}^{2}$. A specific meander arrangement of the nickel-chrome heater provides a homogenous temperature distribution on the heated bridge. This tiny microstructure arrangement has a response of a few microseconds when heated up.

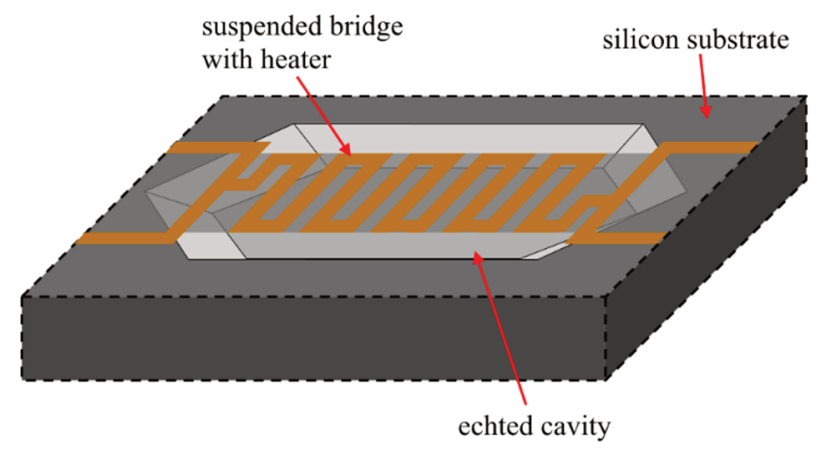

Fig. 3. Simplified setup of thermal MEMS

\subsection{Sensor operation}

The electrical heat source realized with nickel-chrome has a distinct temperature behavior of his electrical resistance. By heating up the structure with current in the submilliampere range, the corresponding temperature rise is several degrees in combination with a resistance change of several $\sim 10 \%$. A fast and highly accurate voltage measurement during an applied constant heating current pulse allows to derive the resistance at any time. The evaluation of the dynamic behavior when applying current pulses is used to get a feedback from the thermal properties of the gases tested.

In Figure 4a the heater current pulse applied is shown. A swichtable precision current source is used to operate the heater. With the current amplitude and the pulse time a fixed amount of energy is feeded into the microsystem. The reaction of the temperature deduced by the resistance measurement is visualized by Fig. 4b. Depending on the thermal conductance of the gas as well as the specific heat transfer, different end values for the temperature respective rise time can be seen. The temperature rise is represented by $\Delta \mathrm{T}$ and the rise time by $\tau$. $4 \mathrm{c}$ and $4 \mathrm{~d}$ illustrate how this different transient reactions are reached. With the assumpten that in both test gas cases the same energy is processed, the gas with higher thermal conductivity reaches a lower level of maximum temperature due to the better conductance of the heat in the gas. Vice versa, the "isolating" gas is responsible that the heat is "blocked" and the temperature amplitude rises. Analog to the thermal conductivity the specific heat capacity has a similar effect. The amount of heat transfered with the pulse in the measurement structure per time unit is identical. For a gas with higher specific heat capacity the rise in temperature is slower than for a gas with lower one. Normalizing the rise time to the end evalue of the temperature plateau enables to conclude on $\mathrm{Cp}$ of the gas mixture.
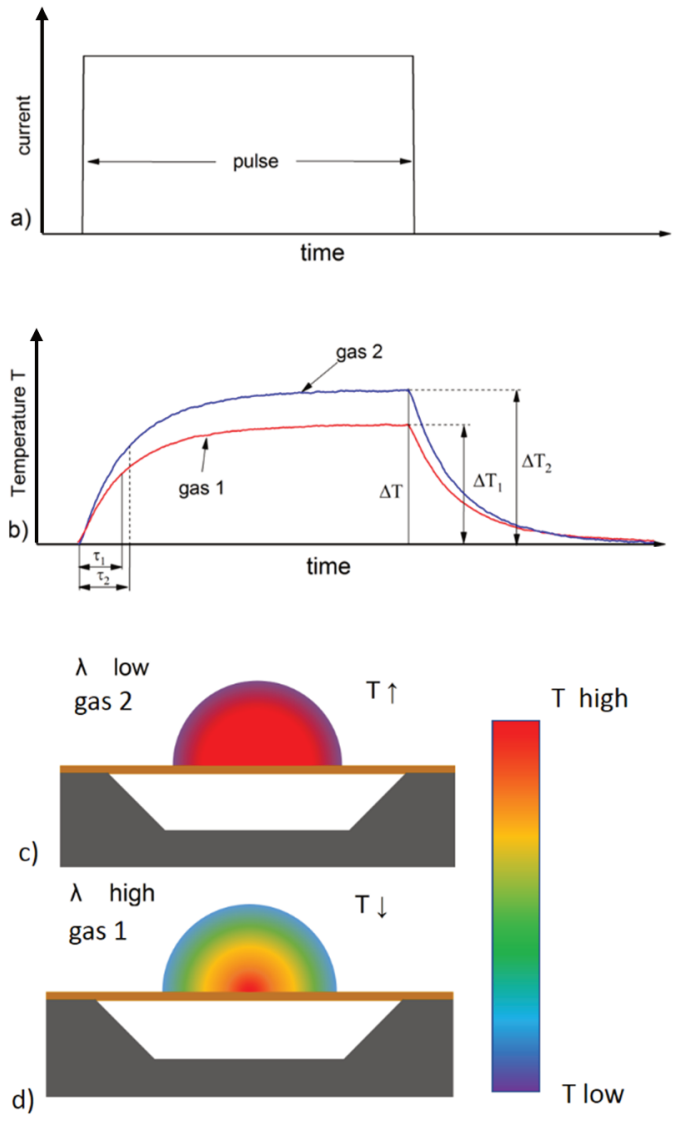

Fig. 4. a) current pulse for heating; b) temperature transient for two different gas species; temperature distribution on top of bridge structure for c) gas 1 and d) gas 2

The heat pulse is chosen to generate only a slight temperature reaction to determine the thermal properties at the tested gas temperature. A second sequence was developed to raise the bridge structure to a constant overtemperature. This overtemperature is heating up the surrounding gas molecules. When the applied heating pulse lasts enough, a small area with permanent higher gas temperature is created. With a third measurement pulse, similar to the first one, the thermal properties of the overheated gas can be derived with the difference that they represent the gas properties at overtemperature with sufficent accuracy. Thermal data for different gas temperatures can be collected with this methode.

\subsection{Correlation model}

Of course, the calorific values can be obtained with a caloric measurement device by gas combustion and measuring the heat release. This is not possible with a simple sensor device at automotive environmental conditions. Besides of optical spectroscopy, a multi-parameter correlation method may be expedient. By measuring other properties of the relevant gas and the use of statistical means a prediction of a target value can be obtained. In general, these properties don not necessarily have to be linked to the parameter of interest, like in our case the thermal properties to the calorific value. If the measurement value gives an adequate gas component distinction, a correlation algorithm 
can achieve good results with sufficient accuracy. The optimization of the algorithm needs a defined set of gases.

Figure 5 shows an overview how the signal flow is handled to get a prediction of combustion relevant gas parameters. Out of the main fractions of the CNG the theoretical values for the thermal conductivity and the specific heat capacity are calculated over temperature. A set of four input parameters was taken for dedicated $\mathrm{CNG}$ gas blends and applied to numerous equation types. The input parameters were joined in a virtual value SCV (sensor correlated value) which is a function of $\lambda(\mathrm{T} 1), \lambda(\mathrm{T} 2), \mathrm{cp}(\mathrm{T} 1)$ and $\mathrm{cp}(\mathrm{T} 2)$, where $\mathrm{T} 1$ represents the gas temperature and $\mathrm{T} 2$ a fixed gas overtemperature. The mathematic correlation with the smallest average deviation for LCV prediction was taken for further steps. In analogy to the LCV the correlation can be done with the methane number or the stoichiometric required Air Fuel Ratio for combustion.

In the following Figure 5 the $\mathrm{SCV}$ as a function of $\lambda(\mathrm{T} 1), \lambda(\mathrm{T} 2), \mathrm{cp}(\mathrm{T} 1)$ and $\mathrm{cp}(\mathrm{T} 2)$ is shown. The good correlation result can be fitted with a quadratic equation. The error between the calculated values and the fit of the single gas is shown in the deviation chart. The deviation is normalized to the LCV of methane. For the investigated gases the theoretically achievable accuracy is better than $\pm 2 \%$.

Like described in the previous section the sensor measures a rise time and a temperature change, derived from the heater resistance, representing the values of $\lambda$ and $\mathrm{cp}$. The substitution of the values with the measured values of $\Delta \mathrm{T}$ and $\tau$ and some small modifications in the equation parameters lead to the following result, when tested with the same gas blends.

With a slight modification of the equation and the inserted values of $\Delta \mathrm{T} 1, \Delta \mathrm{T} 2, \tau 1$ and $\tau 2$ the $\mathrm{LCV}$ prediction ends up in a similar good result, shown in Fig. 9. A linear regression of $\mathrm{LCV}$ based on $\operatorname{SCV}(\Delta \mathrm{T}, \tau)$ is possible. The normalized deviation stayed in an error range of $2 \%$ for the tested gas blends.

\section{Test bench results}

After finding a promising correlation approach and static pre testing, the quality of the correlation result was validated with a dynamic blend testing. Hydrocarbon/inert gas blends, mixed by flow controllers were used at a the test bench. Successively, five identical sensor samples performed an automated test cycle over several minutes. After testing each of the nine test gases, a purge cycle with pure nitrogen and low flow was applied to get defined starting conditions. The result can be seen in Fig. 6 .

It can be seen, that the gas variations caused the same reaction on the calibrated sensor devices with a small part to part variation. The single raw data value showed a noise level of $\sim 1.5 \%$. This value can be reduced significant by averaging the sensor output signals. The sensors also reacted in the same way when changing from gas to purge sequence. For example, gas blend no. 1 lead instantaneously to a steady state, while gas blend no. 7 has a delayed mixing, with a time constant of several $\sim 10$ seconds. The calculated deviation was in the range of $\pm 4 \%$ without the averaging measure. This error is higher than the pure static measurement, but still in an acceptable range. select main CNG compounds

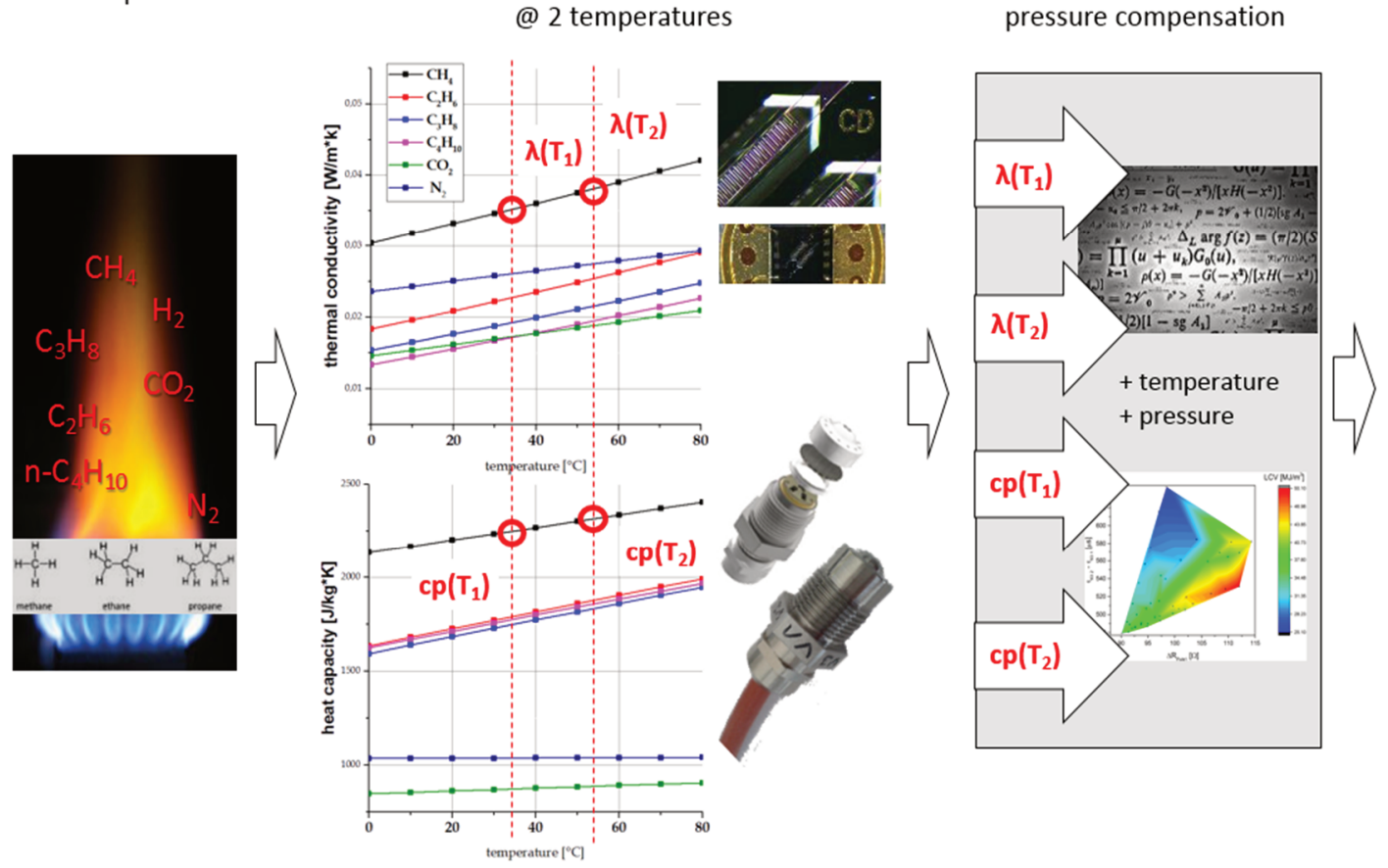

measure thermal properties of gas mixture (heat capacity and thermal conductivity) @ 2 temperatures

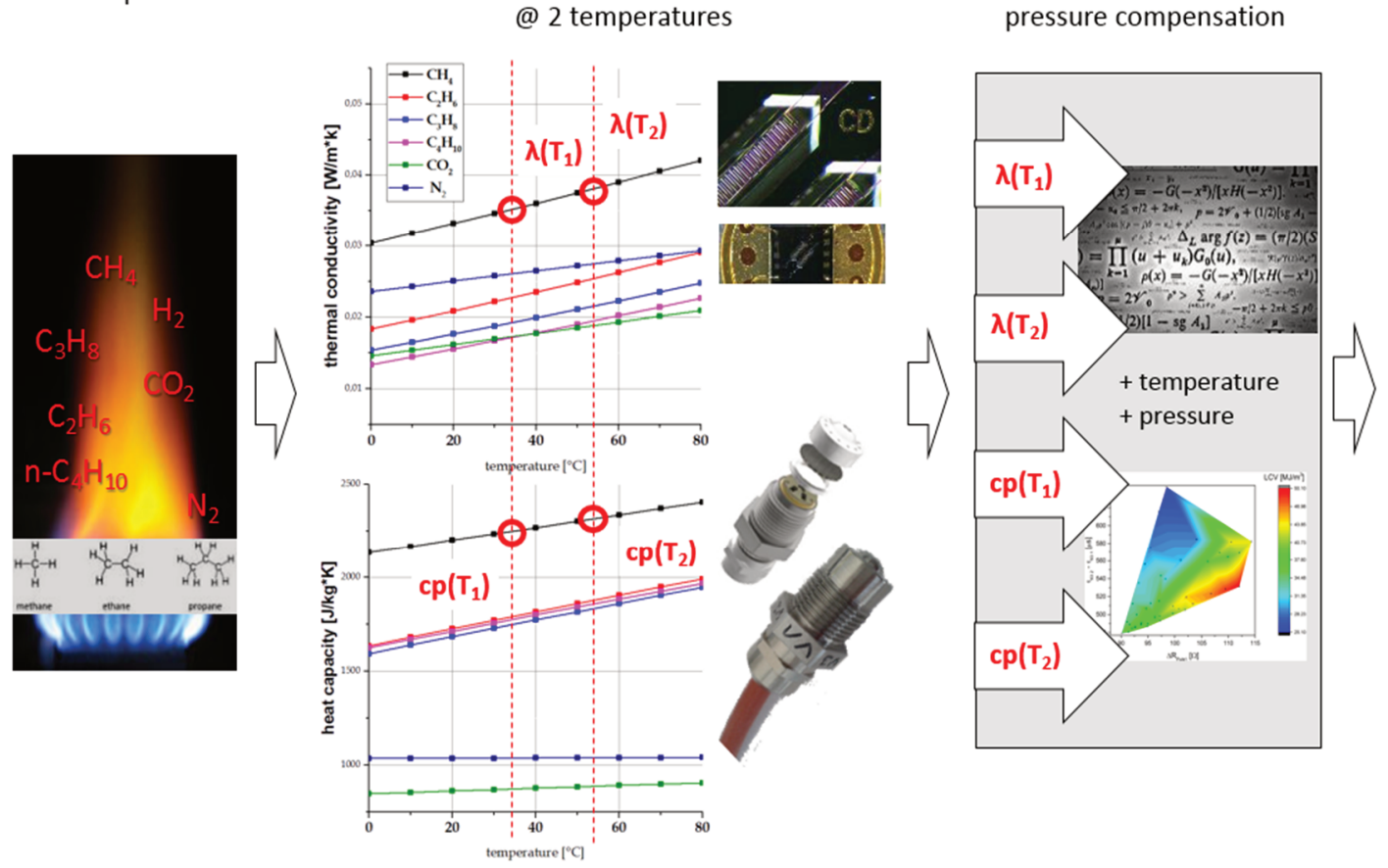

prediction model with sensor inputs and pressure compensation

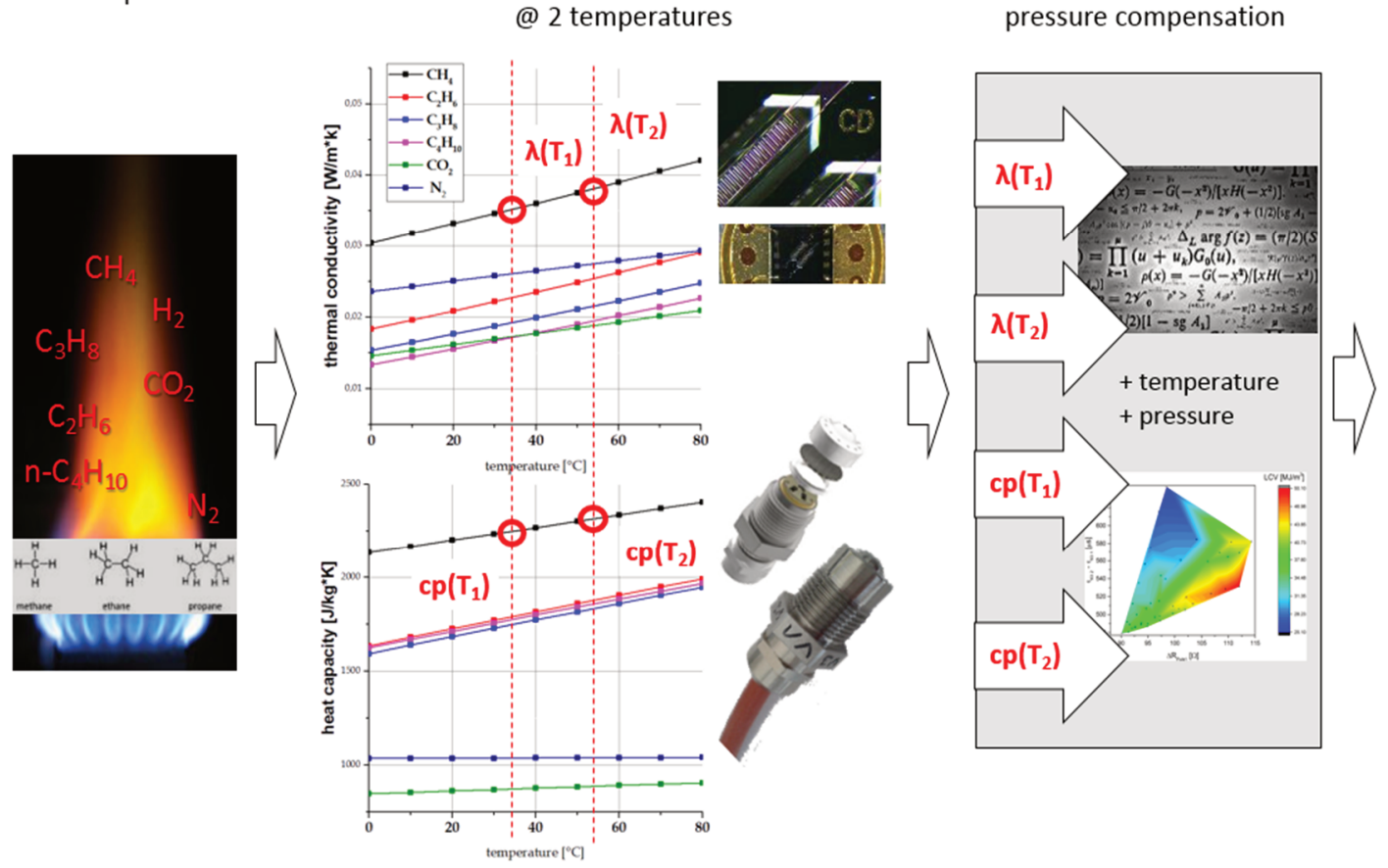

correlated combustion relevant parameters

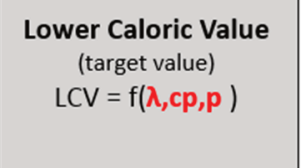

Methane Number (optional)

$M N=f(\lambda, c p, p)$

Air Fuel Ratio (optional)

$A F R=f(\lambda, c p, p)$

Fig. 4. Concept of the signal propagation of a multiple input correlation setup to predict quality values for CNG 

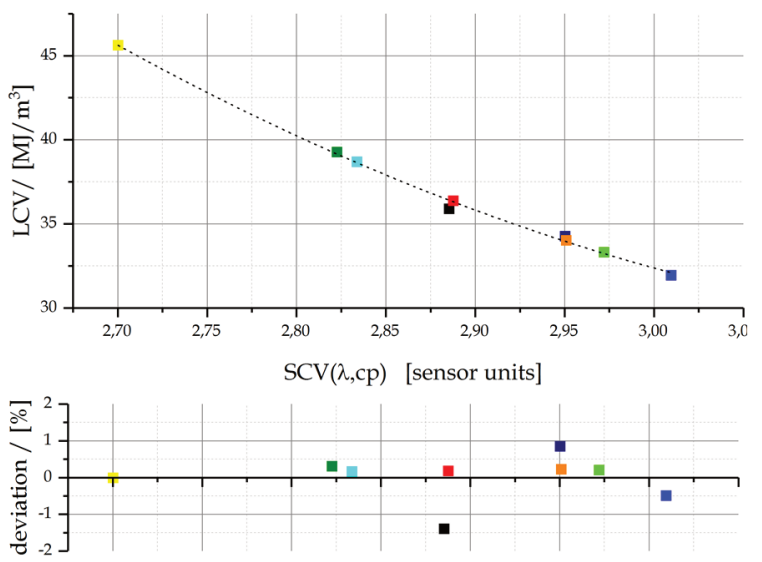

\begin{tabular}{|c|c|c|c|c|c|c|}
\hline \multirow{2}{*}{$\begin{array}{c}\text { Gas } \\
\text { No. }\end{array}$} & \multicolumn{7}{|c|}{ Gas compound [vol\%] } \\
\cline { 2 - 7 } & $\mathrm{CH}_{4}$ & $\mathrm{C}_{2} \mathrm{H}_{6}$ & $\mathrm{C}_{3} \mathrm{H}_{8}$ & $\mathrm{C}_{4} \mathrm{H}_{10}$ & $\mathrm{CO}_{2}$ & $\mathrm{~N}_{2}$ \\
\hline 1 & 100 & 0 & 0 & 0 & 0 & 0 \\
\hline 2 & 71 & 1 & 11 & 0 & 0 & 17 \\
\hline 3 & 83 & 4 & 1 & 0 & 1 & 11 \\
\hline 4 & 81 & 3 & 1 & 0 & 1 & 14 \\
\hline 5 & 91 & 6 & 1 & 1 & 1 & 0 \\
\hline 6 & 79 & 9 & 3 & 7 & 2 & 0 \\
\hline 7 & 84 & 3 & 1 & 1 & 1 & 10 \\
\hline 8 & 90 & 6 & 2 & 1 & 1 & 0 \\
\hline 9 & 85 & 4 & 1 & 0 & 0 & 10 \\
\hline
\end{tabular}

Fig. 5. Result of the theoretical correlation for the lower calorific value over a set of gas blends

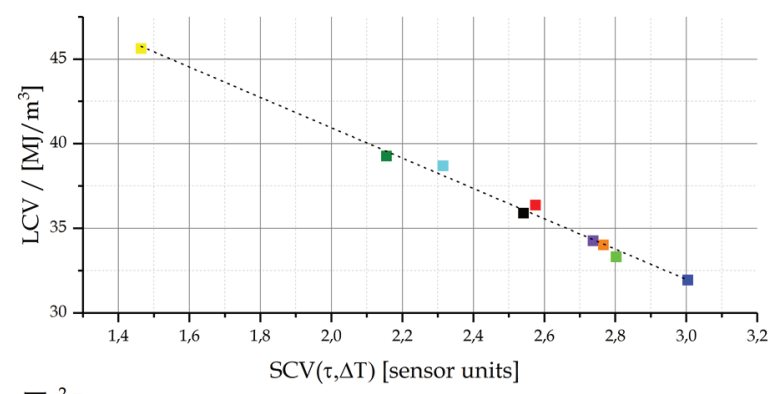

\begin{tabular}{|c|c|c|c|c|c|c|}
\hline \multirow{2}{*}{$\begin{array}{c}\text { Gas } \\
\text { No. }\end{array}$} & \multicolumn{7}{|c|}{ Gas compound [vol\%] } \\
\cline { 2 - 7 } & $\mathrm{CH}_{4}$ & $\mathrm{C}_{2} \mathrm{H}_{6}$ & $\mathrm{C}_{3} \mathrm{H}_{8}$ & $\mathrm{C}_{4} \mathrm{H}_{10}$ & $\mathrm{CO}_{2}$ & $\mathrm{~N}_{2}$ \\
\hline 1 & 100 & 0 & 0 & 0 & 0 & 0 \\
\hline 2 & 71 & 1 & 11 & 0 & 0 & 17 \\
\hline 3 & 83 & 4 & 1 & 0 & 1 & 11 \\
\hline 4 & 81 & 3 & 1 & 0 & 1 & 14 \\
\hline 5 & 91 & 6 & 1 & 1 & 1 & 0 \\
\hline 6 & 79 & 9 & 3 & 7 & 2 & 0 \\
\hline 7 & 84 & 3 & 1 & 1 & 1 & 10 \\
\hline 8 & 90 & 6 & 2 & 1 & 1 & 0 \\
\hline 9 & 85 & 4 & 1 & 0 & 0 & 10 \\
\hline
\end{tabular}

Fig. 6. Result of the theoretical correlation for the lower calorific value over a set of gas blends

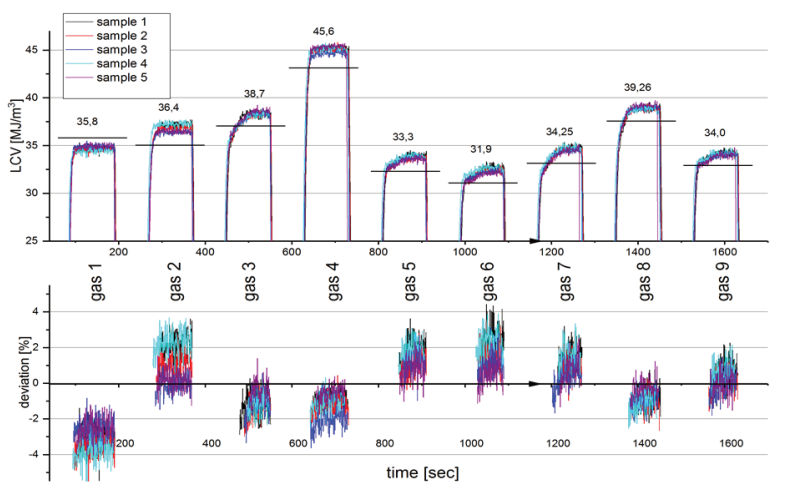

\begin{tabular}{|c|c|c|c|c|c|c|c|c|c|}
\hline \multirow{2}{*}{ Gas } & \multicolumn{10}{|c|}{ Gas blends [vol\%] } \\
\cline { 2 - 11 } & $\mathbf{1}$ & $\mathbf{2}$ & $\mathbf{3}$ & $\mathbf{4}$ & $\mathbf{5}$ & $\mathbf{6}$ & $\mathbf{7}$ & $\mathbf{8}$ & $\mathbf{9}$ \\
\hline $\mathrm{CH}_{4}$ & 100 & 71 & 91 & 79 & 83 & 81 & 84 & 90 & 85 \\
\hline $\mathrm{C}_{2} \mathrm{H}_{6}$ & 0 & 1 & 6 & 9 & 4 & 3 & 3 & 6 & 4 \\
\hline $\mathrm{C}_{3} \mathrm{H}_{8}$ & 0 & 11 & 1 & 3 & 1 & 1 & 1 & 2 & 1 \\
\hline $\mathrm{C}_{4} \mathrm{H}_{10}$ & 0 & 0 & 1 & 7 & 0 & 0 & 1 & 1 & 0 \\
\hline $\mathrm{CO}_{2}$ & 0 & 0 & 1 & 2 & 1 & 1 & 1 & 1 & 0 \\
\hline $\mathrm{N}_{2}$ & 0 & 17 & 0 & 0 & 11 & 14 & 10 & 0 & 10 \\
\hline \multicolumn{10}{|c|}{$\mathrm{T}=23^{\circ} \mathrm{C}, \mathrm{P}=1$ bar abs. } \\
\hline
\end{tabular}

Fig. 7. Testing of synthetic gas blends to predict the lower calorific value

\section{Conclusion}

Measuring the quality of CNG gas is a difficult topic. Direct measurements are not possible due to environmental limitations or are simply to complex and costly to be able entering the automotive market. Therefore a concept study was done with a correlative method based on the thermal properties of the typical single gas components of CNG. The statistical investigations were done with a novel MEMS chip based on a thermal isolated bridge structure. Before a dedicated test cycle was developed to measure the thermal conductance and the specific heat capacity a set of equations was tried and optimised. Parameter for the optimisation was the minimum error level on the lower calorific value for the entirety of defined test blend set.

After fixing a set of equations a comparison of the theoretical calculated accuracy levels was done with the corresponding test bench measurement. The basic investigation showed for the calculated as for the measured test blends similar accuracy levels of less than $2 \%$. 
To have a first overview about the principle robustness and the performance spread over different samples a test sequence with nine test blends at low flow was done. A fluctuation on the signal could be observed, which can be optimised by signal filtering and averaging. Without this optimisation the error was in the range of $4 \%$.

For sure the measurement has some weaknesses compared to a colorimeter. However the low effort for electron- ic and the affordable miniaturized sensing element allow to get a solution which could fulfill the requirements of an automotive application. The first investigations have shown that a calorific value prediction with an accuracy level in the lower percentage range is possible. This information before engine start could be sufficient for a defined and save engine start in a monovalent CNG car.

\section{Bibliography}

[1] MESCHEDE, D., Gerthsen Physik, Springer Berlin Heidelberg, 2015.

[2] MASON, E.A., SAXENA, S.C. Approximate Formula for the Thermal Conductivity of Gas Mixtures. The Physics of Fluids. 1958, 361-369.

[3] CERBE, G., LENDT, B. Grundlagen der Gastechnik, München: Hanser. 2017, 68.

[4] CZICHOS, H. Die Grundlagen der Ingenieurwissenschaften, Springer Berlin Heidelberg. 2000, B83.

[5] POLEZHAEV, Y.V, Thermal Conductivity in: A-Z Guide to Thermodynamics. Heat and Mass Transfer and Fluids Engineering. DOI:10.1615/AtoZ.t.thermal_conductivity.

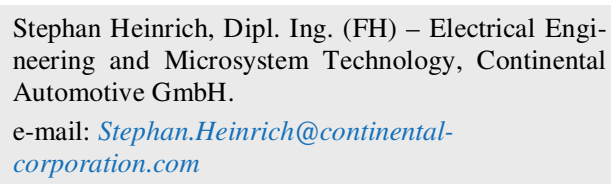

[6] VAN BASSHUYSEN, R. Erdgas und erneuerbares Methan für den Fahrzeugantrieb. Springer Fachmedien Wiesbaden, 2015, 70.

[7] Verein Deutscher Ingenieure VDI-Gesellschaft and Verfahrenstechnik und Chemieingenieurwesen (GVC), VDI-Wärmeatlas, Springer Berlin Heidelberg, 2006, Da16, Da17, Da25, DOI:10.1007/978-3-540-32218-4.

[8] CARTELLIERI, W., PFEIFER, U. Forschungsberichte Verbrennungskraftmaschinen - Erweiterung der Energieerzeugung durch Kraftgase Teil 3 - Untersuchungen zur Übertragbarkeit der am CFR-Motor gefundenen Ergebnisse auf andere Motoren - Gültigkeitsbereich der Methanzahl, 1971.

Marcus Hein, DEng. - Mechatronics, Continental Automotive $\mathrm{GmbH}$.

e-mail: Markus.Hien@continental-automotive.com

Josef Muggli, B.Sc. (FH) - Sensor and Analytics,

Continental Automotive GmbH.

e-mail: Josef.2.Muggli@continental-

corporation.com 